\title{
Light Scattering in KDP Crystals and Relaxation Oscillations of Diffusion Layer in Crystal Growth from Solutions
}

\author{
Vladimir Bredikhin*, Galina Galushkina and Stanislav Kuznetsov \\ Institute of Applied Physics of the Russian Academy of Sciences, Nizhny Novgorod, Russia
}

\begin{abstract}
The problem of achieving high optical quality of crystals (KDP monocrystals in particular) grown from solutions under conditions of periodic feeding is considered. The experimental data obtained for rapid profiled growth of KDP crystals have demonstrated a pronounced dependence of crystal quality (light scattering) on feeding parameters: period and duty cycle of feeding, feeder height above the growing surface, and feeding intensity. The theoretical analysis has shown that these effects can be explained within the framework of diffusion layer relaxation at periodic feeding in the diffusion-kinetic regime of crystal growth.
\end{abstract}

Keywords: Solution crystal growth, rapid profiled growth, KDP crystal, periodic feeding, diffusion layer.

\section{INTRODUCTION}

High optical quality of crystals (specifically of KDP monocrystals) grown from solution is still an important problem. It is closely connected with the development of new efficient techniques [1-3] of crystal growth and increased demands to the quality of crystals to be used, for example, in high-power lasers [4-6]. The main quality indexes, such as optical transparency, optical homogeneity, and laser breakdown threshold are related to growth perfection. Indeed, in perfect growth of crystal surface due to the motion of a regular train of elementary steps generated by a dislocation, the height of the elementary steps is, as a rule, $\leq 1 \mathrm{~nm}$. Therefore, only very small inclusions (actually, only point defects of impurity type) can be trapped. Any distortion of step train regularity results in formation on the surface of structures with total height from several nanometers up to tens of micrometers, which enables trapping microinclusions of a larger size, hence, increasing light scattering in the crystal. Irregular density of elementary steps leads to inhomogeneous trapping of impurities and mechanical stresses in the crystal, which, in turn, result in optical nonuniformity and a decrease of optical stability.

Meanwhile, solution crystal growth by a regular train of elementary steps occurs very seldom, at least, in large-size crystals. The point is that in the presence of a diffusion layer, that is always present in crystal growth from solutions, a regular train is unstable even if the diffusion layer is homogeneous and stationary [7]. If the diffusion layer is inhomogeneous or there occurs density modulation of the elementary steps generated by the dislocation source (due to modulation of supersaturation), the distance from the step source to the visible step bunches is smaller [7]. It is logical to assume that developing instability of a regular train of elementary steps results in the formation of step bunches

*Address correspondence to this author at the Institute of Applied Physics of the Russian Academy of Sciences, Nizhny Novgorod, Russia;

Tel: +7 831 4164979; Fax: +7 831 4363792; Emails: bredikh@appl.sci-nnov.ru, vladimirbredikhin@yahoo.com
[7-9] ${ }^{1}$. The motion of step bunches was investigated experimentally and theoretically in Refs. [8, 9], where it was demonstrated that the bunch height, period, and speed are determined by the relationship between the crystal growth rate and intensity and uniformity of feeding of the growing surface.

The model of a nonstationary diffusion layer of preset thickness proposed in $[8,9]$ allows estimating parameters of bunches and thus predicting the degree of crystal growth perfection. However, crystal growth in real conditions is much more complicated: the solution flow velocity and the diffusion layer thickness are nonuniform over the growing surface and vary in time. In the high-rate profiled growth technology [1, 2], the solution is fed onto the growing surface through a feeder periodically moving along the surface, whereas in the high-rate polyhedral technology [3], the crystal rotates reversibly, following a special program. Besides, a crystal usually does not grow by a single growth center and, in the technology described in [3], by a single face. Therefore, it is currently impossible to accurately calculate growth perfection. Model experiments and theoretical studies of the role of various growth parameters on the quality of grown crystals are needed for this.

The objective of the present paper is to examine the influence of periodic feeding and relaxation oscillations of the diffusion layer on crystal quality. The scattering of light is taken to be the parameter of crystal quality. The more rough the growth bunches, the more probable trapping of suspended particles (or solution inclusions) by the crystal is. The roughness, in turn, depends on intensity and uniformity (in space and time) of the growing surface feeding. That is why light scattering in crystals not only characterizes crystal quality, but also indicates adequate growth conditions at a chosen growth rate. Light scattering is recorded much simpler compared to other characteristics of crystal quality, such as, e.g., absorption spectra or laser breakdown

\footnotetext{
${ }^{1}$ Several growth sectors (including vicinal ones) giving rise to the corresponding defects in the crystal can be also considered as disturbance of a regular train of steps.
} 
threshold. Therefore, we have chosen the value of light scattering as the main criterion of crystal quality and growth "roughness".

\section{EXPERIMENT}

\subsection{Growth Experiments}

A model corresponding to the high-rate profiled growth technology $[1,2]$ is chosen as the basic model of crystal growth. The schematic of the setup for growing crystals by a single face fed by a supersaturated solution through a periodically moving feeder $[1,2]$ is shown in Fig. (1), and the scheme of the solution flow directed to the crystal face in this setup is presented in Fig. (2). This crystal growth scheme is simplest for studies, as it allows varying parameters, such as flow intensity, distance between the feeder and the growing surface, solution temperature, and others almost independently.

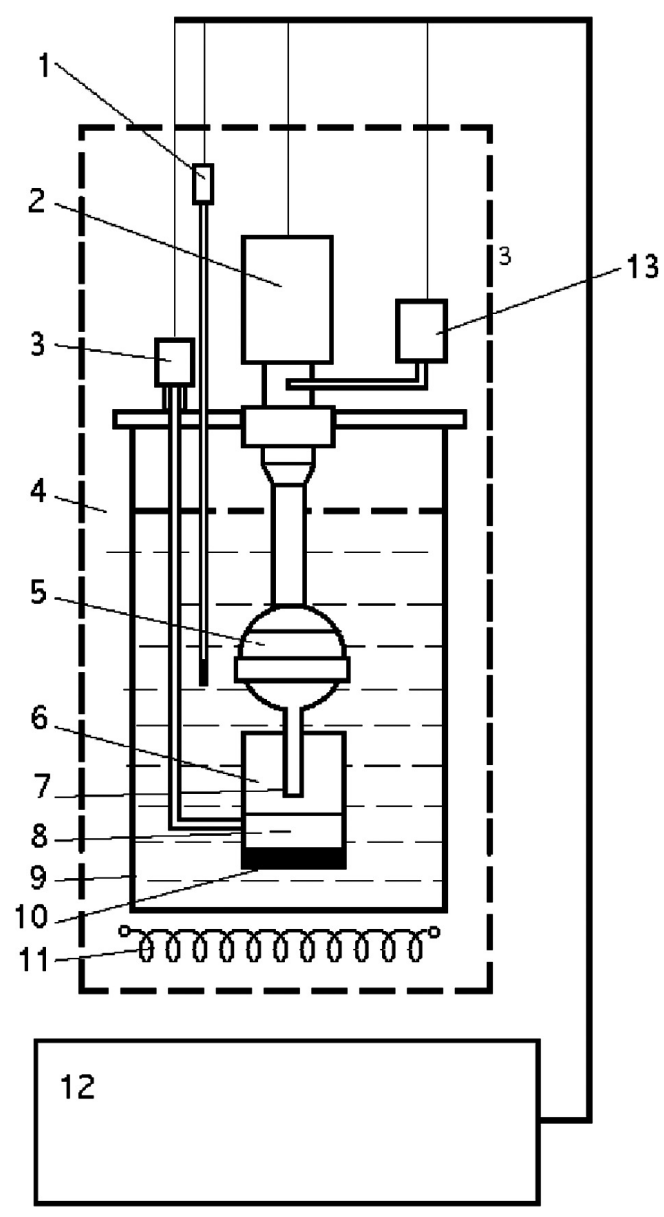

Fig. (1). The scheme of the crystallizer for rapid oriented crystal growth: 1 - contact thermometer; 2 - pump driver; 3 - chamber moving device ; 4 - thermostat; 5 - pump; 6 - growth chamber; 7 - feeder; 8 - growing crystal; 9 - vessel; 10 - seed; 11 - heater; 12 - control block; 13 - feeder moving device.

A setup of high-rate profiled growth with the crosssection of a growth chamber (and of a grown crystal) of $43 \times$

${ }^{2}$ Note that the classical crystallographic methods of assessing crystal perfection (x-ray topography, rocking curve, and others) are of little use for large-size high-quality (laser quality) single crystals because of their low sensitivity.
$43 \mathrm{~mm}^{2}$ was used in the study $[7,10,11]$. The experiments were carried out for growing KDP crystals by the (101) face. To simplify measurements and improve reliability of results the growth solution had been doped with polymer microsuspensions $\leq 1 \mu \mathrm{m}$ in diameter. An average normal growth rate of about $0.5 \mathrm{~mm} / \mathrm{h}$ was sustained. A series of experiments were performed, in which the value of solution flow, distance between the feeder and the growing surface (these parameters were varied independently in one growth experiment), and widths $(4.5,2.5,8$, and $16 \mathrm{~mm})$ of the feeder slits (in various experiments) were varied. The swing period $2 T$ of the feeder was $2 \mathrm{~s}$.

\subsection{Light Scattering Measurements}

Light scattering was measured ex situ in samples with polished end faces in a He-Ne laser beam at $\lambda=0.63 \mu \mathrm{m}$ at an angle of about $27^{\circ}$ to the laser beam in relative units with respect to the light scattering value in the "reference" sample made of TF-111 optical glass. This permitted sustaining the same unit of measuring light scattering for a long time. Light scattering was measured both photoelectrically and visually (using the method of aligning the scattered light from the reference and studied samples by calibrated neutral light filters). In both cases, measures were taken to exclude light scattering at the input and output end faces.

Various vicinal sectors of the growth hillock of the (101) face of a KDP crystal have different slopes and, hence, different ability to trap inclusions [12], which results in nonuniform distribution of light scattering intensity in the crystal. The maximum value of light scattering in the studied region, i.e. in the layer $5 \div 10 \mathrm{~mm}$ thick grown under fixed conditions, was taken to be the value of light scattering in this region.

\section{EXPERIMENTAL RESULTS}

The diagrams in Fig. (3) show the light scattering distribution in two samples grown by means of a feeder having width $\approx 4 \mathrm{~mm}$. The feeder height above the crystal was 3-4 mm. It is seen in Fig. (3) that light scattering in the crystals grown using this feeder $(/ / L \sim 0.1)$ greatly depends on the feeding intensity, and the minimum value (corresponding to optical quality crystals) is achieved at the flow velocity $\sim 150 \mathrm{~cm} / \mathrm{s}$, which conforms with an average flow rate of the solution of about $13 \mathrm{~cm} / \mathrm{s}$ (or $\sim 0.8$ $1 / \mathrm{min} \cdot \mathrm{cm}^{2}$ ).

To study the possibilities of reducing the needed solution flow consumption for the same crystal quality, we carried out experiments on crystal growth using $2.5,4,8$, and I6 mm feeders. In those experiments, both the flow velocities and the feeder height $H$ (Fig. 2) above the crystal were varied in turn. The light scattering in these crystals as a function of height $H$ are plotted in Fig. (4). It is interesting that the dependences of light scattering on height are opposite in the experiments with $2.5 \mathrm{~mm}$ and $16 \mathrm{~mm}$ feeders, and in the experiment with a $8 \mathrm{~mm}$ feeder the light scattering $(\sim 0.5)$ is almost independent of $H$. In the latter case, the light scattering weakly depends on the number of pump revolutions as well.

The feeding parameters (pump revolutions, flow velocity, average flow rate per $\mathrm{cm}^{2}$ ) needed for obtaining satisfactory 


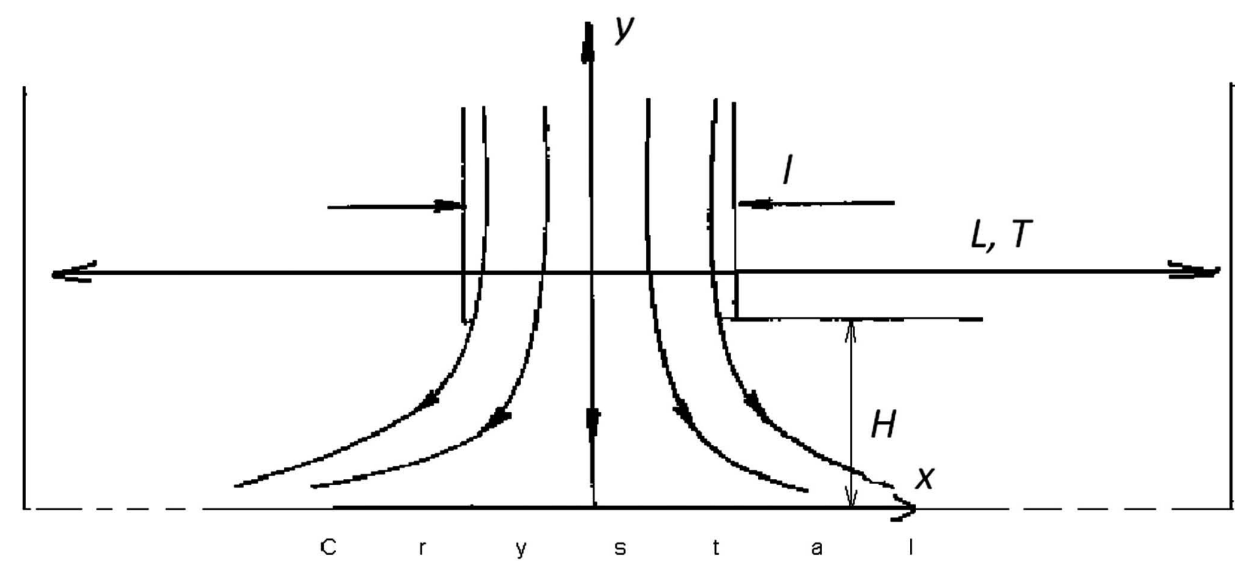

Fig. (2). The scheme of the solution flow directed to the crystal face $(l$ is feeder width, $L$ and $2 T$ are the amplitude and period of feeder oscillation, and $H$ is the distance between the feeder and the growing surface.

results (light scattering is $\sim 0.3$ ) using various feeders are listed in Table 1. It should be kept in mind that those results were obtained in different experiments, in which other parameters, such as seed, filtration membrane, etc. could change automatically. From Table $\mathbf{1}$ it follows that the minimal flow consumption was observed for the wide feeder of $16 \mathrm{~mm}(l / L \approx 0.4)$.
Pump, rpm Light scattering

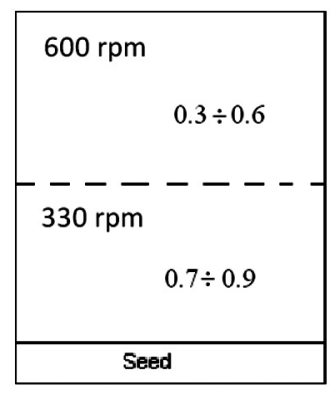

\#1
Pump, rpm Light scattering

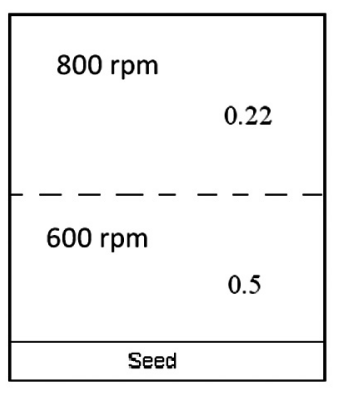

\#2
Fig. (3). Light scattering in two crystals grown at various pump revolutions. The pump rotation velocity of $330 \mathrm{rpm}$ corresponds to the velocities of the solution outflow from the feeder equal to $50 \mathrm{~cm} / \mathrm{s}, 600 \mathrm{rpm}-95 \mathrm{~cm} / \mathrm{s}, 800 \mathrm{rpm}-135 \mathrm{~cm} / \mathrm{s}$. The feeder slit width is $4 \mathrm{~mm}$. Light scattering is given in relative units of the light scattering value in the reference sample made of F-111 optical glass.

\section{RELAXATION OF RELATIVE SUPERSATURATION ON THE GROWING SURFACE AT PERIODIC MOTION OF A FEEDER}

Periodic motion of a feeder leads to a periodic time dependence of relative supersaturation $\sigma$ on the growing surface and to some reciprocal nonuniformity of the average value of relative supersaturation over the growing surface (along the $x$-coordinate (Fig. 2)). The dependence of supersaturation $\sigma$ on $x$ and $t$ is rather complicated. This problem was considered in Ref. [13] in the approximation of a constant value of supersaturation gradient with respect to $y$ $\left(\left.\frac{\partial \sigma}{\partial y}\right|_{y=0}=\right.$ const $)$. Attention should be paid here to the periodic time dependence of relative supersaturation on the growing surface. This dependence implies fulfillment of the third-type boundary conditions on the growing surface: $\left.D C_{0} \frac{\partial \sigma}{\partial y}\right|_{y=0}=\rho \sigma \beta n h(D$ is diffusion coefficient, $\rho$ is crystal

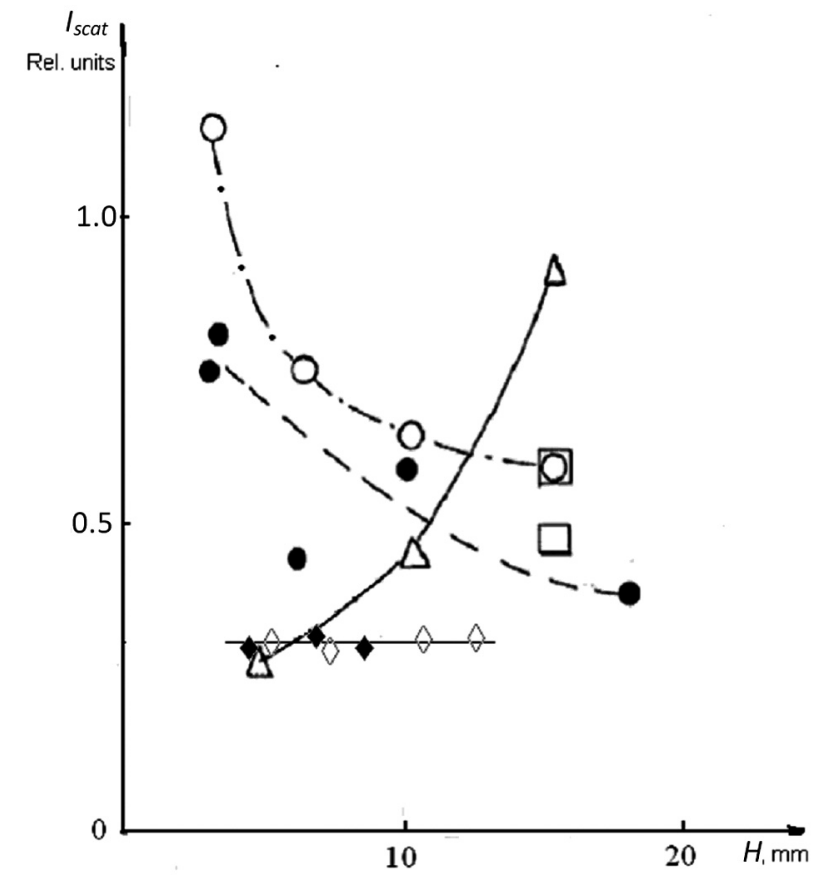

Fig. (4). Light scattering $I_{\text {scat }}$ in DKDP single crystals $(43 \times 43$ $\mathrm{mm}^{2}$, (101)) vs feeder height $\mathrm{H}$ above crystal: $\Delta$ and $\square$ for $16 \mathrm{~mm}$ feeder width, $\bullet$ and $\circ$ for $2.5 \mathrm{~mm}$ feeder width, $\Delta$ and $\circ$ for the pump rotation rate of $650 \mathrm{rpm}$ (flow velocities $20 \mathrm{~cm} / \mathrm{s}$ and 128 $\mathrm{cm} / \mathrm{s}$, respectively), $\square$ and $\bullet$ for $900 \mathrm{rpm}$ (flow velocities $45 \mathrm{~cm} / \mathrm{s}$ and $180 \mathrm{~cm} / \mathrm{s}$, respectively), and $\diamond$ for the feeder width of $8 \mathrm{~mm}$, $\diamond$ is the pump rotation rate of $700 \mathrm{rpm}$ (flow velocity $70 \mathrm{~cm} / \mathrm{s}$ ), $\downarrow$ is the pump rotation rate of $500 \mathrm{rpm}$ (flow velocity $45 \mathrm{~cm} / \mathrm{s}$ ). 
Table 1. The Feeding Parameters Needed for Obtaining Satisfactory Results using Various Feeders

\begin{tabular}{|c|c|c|c|}
\hline Feeder Width & Pump Revolutions, rpm & Flow Speed cm/s & Flow Consumption $\left(\mathbf{l} / \mathbf{m i n} \cdot \mathbf{c m}^{2}\right)$ \\
\hline \hline 2.5 & 950 & 190 & $\approx 0.6$ \\
\hline 4 & 800 & 135 & $\approx 0.7$ \\
\hline 8 & $500 \div 700$ & $45 \div 70$ & $\approx 0.5 \div 0.8$ \\
\hline
\end{tabular}

density, and $C_{0}$ is solution concentration). Growth rate $R$ is related to step propagation as $R=h n v$, the velocity $v$ of elementary steps is related to the relative solution supersaturation $\sigma_{x}$ on the surface as $v=\beta \sigma_{x}$, where $\beta$ is kinetic step coefficient and $h$ is step height $[14,8,9])$.

Parameters of this periodic dependence will be estimated in the diffusion layer approximation [8, 9, 13]. This approximation is possible for crystal growth from solutions, as the Schmidt number characterizing the ratio of the thicknesses of the hydrodynamic boundary layer and of the mass transfer layer $\delta$ is much more than unity in liquids [8, 9, 13]. In this approximation, the mass transfer onto the growing surface is described as diffusion in an immobile layer from the boundary (relative supersaturation on which is equal to relative supersaturation in liquid bulk $\sigma_{\infty}$ ) at distance $\delta$ from the growing surface. To make estimates we need to know thicknesses $\delta$ and relaxation times $\tau$ of the diffusion layer and their dependence on solution flow velocity. The geometry of the used growth devices is rather complicated; therefore, it is difficult to calculate the above quantities accurately. However, we can use for estimates the magnitudes of thicknesses $\delta$ calculated for a tangential solution flow on a semi-infinite plane [13] and data for $\delta$ and $\tau$ in the case of a moving feeder in the form of an infinite slot [13] (Fig. 2).

Let the velocity $U$ of the outflow from the feeder vary in time by the law shown in Fig. (5). The relative supersaturations $\sigma_{l}$ and $\sigma_{2}$ correspond to relative supersaturations for continuous solution flows with maximum and minimum velocities $U_{\max }$ and $U_{\min }$, and $\delta_{\min }$ and $\delta_{\max }$ are the corresponding diffusion layer thicknesses $\delta$. The solution supersaturation on the surface will vary by the law represented qualitatively in Fig. (5). Let us find the values of maximum $\sigma_{\max }$ and minimum $\sigma_{\min }$ with respect to relative supersaturations and the duty cycle of feeding $s=t_{f} / T$. We assume for brevity sake that $\sigma_{\infty}=1$. From Fig. (5) we find

$\delta_{\text {min }}=\delta_{2}+\left(\delta_{\max }-\delta_{2}\right) \exp \left(-\left(T-t_{f}\right) / \tau_{2}\right) ;$

$\delta_{\max }=\delta_{1}+\left(\delta_{\min }-\delta_{1}\right) \exp \left(-t_{f} \tau_{1}\right)$,

where $\tau_{1}$ and $\tau_{2}$ are, respectively, the rise and the decay times of relative supersaturation $\sigma, T$ is period, and $t_{f}$ is feeding duration.

Then we find $\delta_{\max }$ and $\delta_{\min }$ :

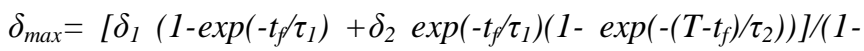
$\left.\exp \left(-\left(T-t_{f}\right) / \tau_{2}\right)-t_{f}\left(\tau_{1}\right)\right)$;

$\delta_{\min }=\delta_{2}+\left(\delta_{\max }-\delta_{2}\right) \exp \left(-\left(T-t_{f}\right) / \tau_{2}\right)$.

In the case of an oscillating feeder $t_{f}=T l / L$, where $T$ is the oscillation half-period, $l$ is feeder width, and $L$ is feeder oscillation amplitude.
In the approximation of linear growth kinetics, supersaturation on the surface $\sigma$ is equal to
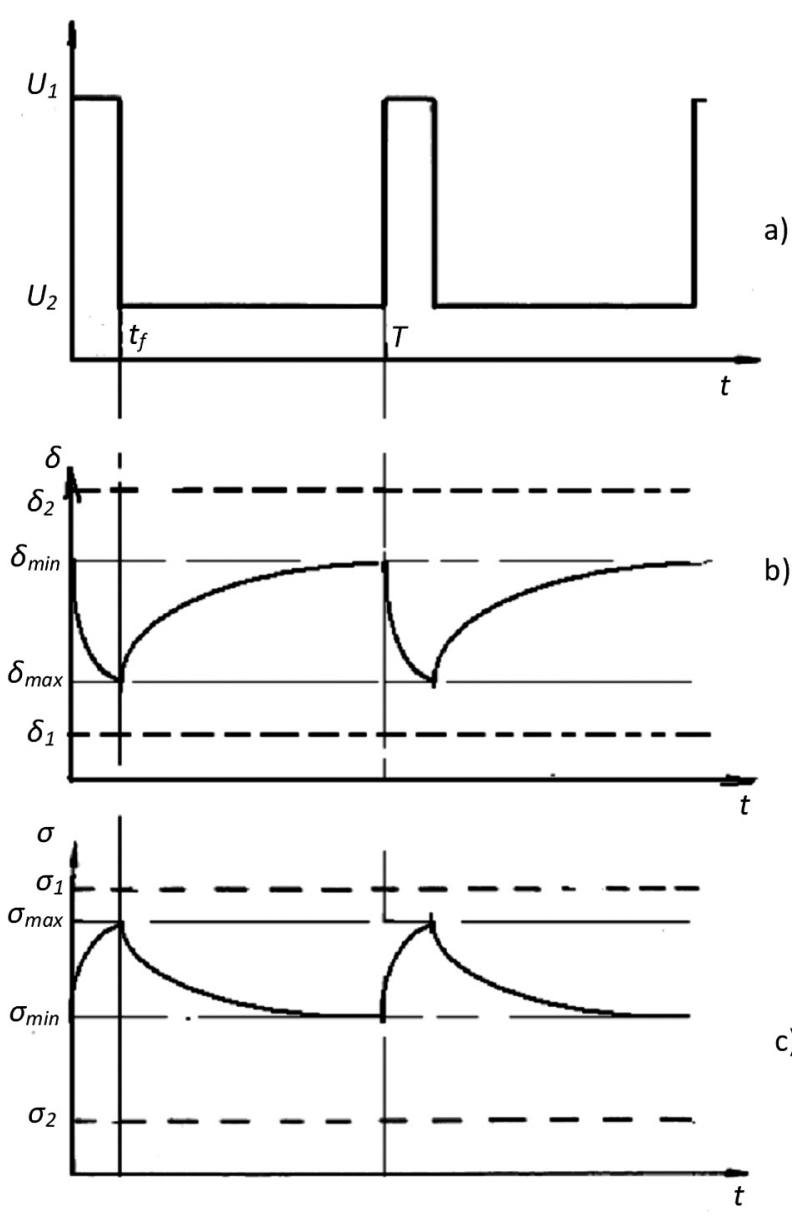

Fig. (5). Flow velocity (a), diffusion layer thickness (b), and relative supersaturation $\sigma(\mathbf{c})$ on the growing crystal surface vs time $t$ at periodic feeding.

$\sigma=\sigma_{\propto} /\left(1+b \rho \delta / D C_{0}\right)$,

where $D$ is diffusion coefficient, $b$ is the kinetic coefficient of face growth, $\rho$ is crystal density, and $C_{0}$ is the concentration of saturated solution.

According to the measurements [12] made in a stationary flow, the operating mode close to the kinetic one sets in at $U^{*} \sim 20 \div 30 \mathrm{~cm} / \mathrm{s}$. Therefore, we assume for definiteness that at this speed $\left(U^{*} \sim 30 \mathrm{~cm} / \mathrm{s}\right)$ "kinetics" of the operating mode is about $90 \%\left(\sigma=0.9 \sigma_{\infty}\right)$. From Eq. (3) we find that in this case $b \rho \delta / D C_{0}$ is equal approximately to 0.11 . 
Since the diffusion layer thickness $\delta$ is inversely proportional to $U^{2}[12,13]$, for the constant flow velocity $U=30 n^{2} \mathrm{~cm} / \mathrm{s}$, we have $b \rho \delta_{1} / D C_{0}=0.11 / n$, whereas for the flow velocity $U=30 \mathrm{n}^{2} / \mathrm{m}^{2} \mathrm{~cm} / \mathrm{s}, b \rho \delta_{2} / D C_{0}=0.11 \mathrm{~m} / \mathrm{n}$.

It follows from Ref. [13] that for the flow velocity ratio $U_{1} / U_{2} \sim 10$ (thus, $\left(\delta_{\min } / \delta_{\max }\right)^{2} \sim 10$ ), the relaxation time $\tau$ in the transition from the initial diffusion layer $\delta_{i}$ to the final diffusion layer $\delta_{f}$ is described by the following relation

$\tau D / \delta_{i}^{2} \approx 0.22\left(\delta_{i} / \delta_{f}\right)^{-2}$,

from which $\left(m^{2} \approx 10\right)$ one can find for $\tau_{1}$ and $\tau_{2}$ :

$\tau_{1}=0.22 \delta_{1}{ }^{2} / D=0.22 \delta_{0}{ }^{2} / n^{2} D ; \quad \tau_{2}=0.22 \delta_{2}{ }^{2} / D=0.22$ $\delta_{0}^{2} m^{2} / n^{2} D$.

For the velocity of the outflow from the feeder of about $30 \mathrm{~cm} / \mathrm{s}$, typical sizes of feeder width, and distance to the crystal of about $1 \mathrm{~cm}, \delta_{0} \approx 0.003 \mathrm{~cm}$; hence we can obtain numerically the following values for $\tau_{1}$ and $\tau_{2}$ (in seconds):

$\tau_{1} \approx 0.22 / \mathrm{n}^{2} ; \tau_{2} \approx 0.22 \mathrm{~m}^{2} / \mathrm{n}^{2}$.

The values of $\sigma_{\max }=\sigma_{\infty} d\left(1+b \rho \delta_{\max } / D C_{0}\right), \sigma_{\min }=\sigma_{\alpha d} /(1+$ $\left.b \rho \delta_{\min } / D C_{0}\right)$, and the relative oscillation amplitude of relative supersaturation

$d=\left(\sigma_{\max }-\sigma_{\min }\right) / \sigma_{\max }$

were calculated numerically by Eqs. (1) - (7). The results of the calculations are presented in Figs. (6-9). Hereinafter, to simplify further analysis and comparison with results of the experiment, the relative value of flow velocity $n$ will be represented in the form $n^{2}=p / s$, where $s$ is relative feeder width $s=l / L$, and $p$ is the relative value of flow velocity. Thus, $q=n^{2} l=p L$ is the total value of the flow supplied by the pump to the crystal relative to the total flow incident on the crystal with speed $30 \mathrm{~cm} / \mathrm{sec}$. The maximum $\sigma_{\max }$ (a and c) and minimum $\sigma_{\min }$ (b and d) values of relative supersaturation $\sigma$ versus relative slit width $s=l / L$ for parameter $n^{2}=0.5 / s$ and constant velocity ratio $m=3$ are plotted in Fig. (6). The feeding half-periods are $1 \mathrm{~s}$ ( $\mathrm{a}$ and $\mathrm{b}$ ) and

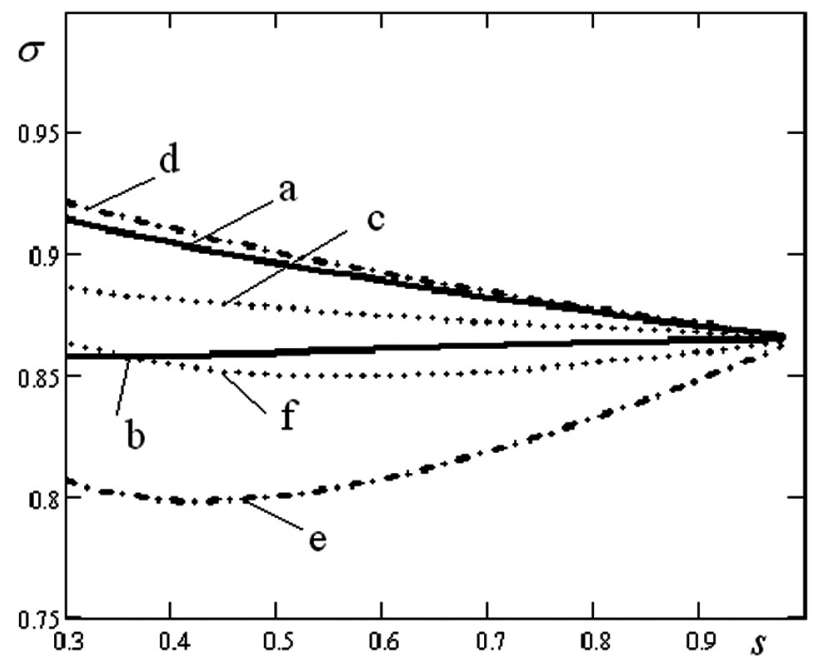

Fig. (6). Maximum $\sigma_{\max }$ (a and d), minimum $\sigma_{\min }$ (b and e) and mean $\sigma_{\text {mean }}(\mathbf{c}$ and $\mathbf{f})$ values of relative supersaturation $\sigma$ vs relative feeder width $s=l / L$ for $n^{2}=0.5 / s$ and constant velocity ratio $m=3$. Feeding half-periods are $1 \mathrm{~s}(\mathbf{a}, \mathbf{b}$ and $\mathbf{c})$ and $4 \mathrm{~s}(\mathbf{d}, \mathbf{e}$ and $\mathbf{f})$.
$3 \mathrm{~s}$ (c and d); c and d are the corresponding curves for mean supersaturation $\sigma_{\text {mean }}$. The relative oscillation amplitude of supersaturation $d$ is plotted in Figs. (7-9) as a function of different parameters: $d$ versus relative flow intensity $p$ for $s=0.1$ (Fig. 7a) and for $s=0.2$ (Fig. 7b); $d$ versus feeder width $s=l / L$ for feeding half-period $T$ equal to $1 \mathrm{~s} \mathrm{(Fig.} \mathrm{8a)} \mathrm{and} \mathrm{to}$ $2 \mathrm{~s}$ (Fig. 8b); $d$ versus feeding half-period $T$ for relative feeder width $s=0.1$ (Fig. 9a) and for $s=0.4$ (Fig. 9b). In all cases the value of $m$ is assumed to be independent of $s=l / L$ and equal to 3 . For the cases depicted in Figs. $(6,8$ and 9$)$ the solution flow per growing surface unit $Q=U \cdot / / L$ is taken to be assigned and equal to $15 \mathrm{~cm} / \mathrm{s}$ (which is close to experimental values), i.e., in this case $n^{2} \sim 0.5 \mathrm{l} / \mathrm{L}$.

\section{DISCUSSION AND RESULTS}

As follows from the results of the theoretical analysis of the maximum and minimum supersaturations, the relative oscillation amplitude depends significantly on growth

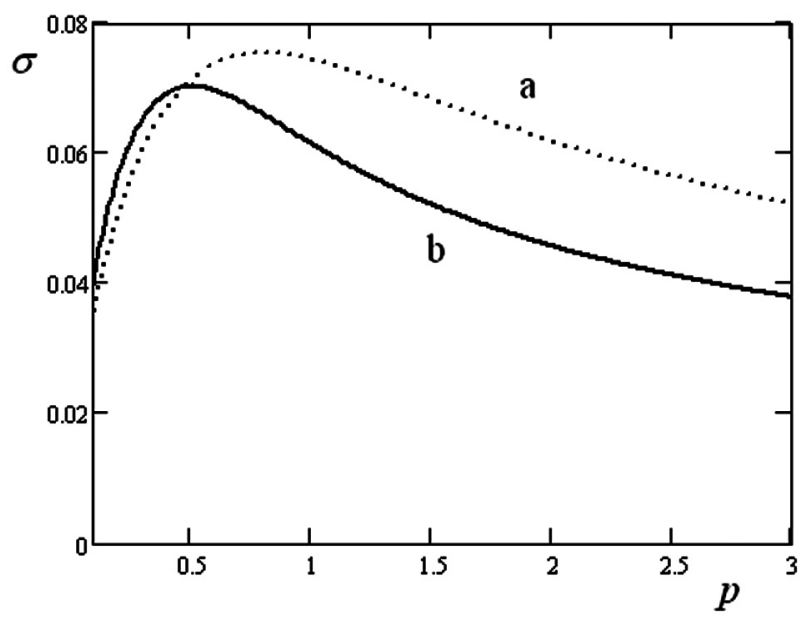

Fig. (7). Oscillation amplitude $d$ of relative supersaturation vs feed power $p$ for feeding half-period $T$ equal to $1 \mathrm{~s}, m=3$, relative feeder width $s=0.1$ (a) and $s=0.2(\mathbf{b})$.

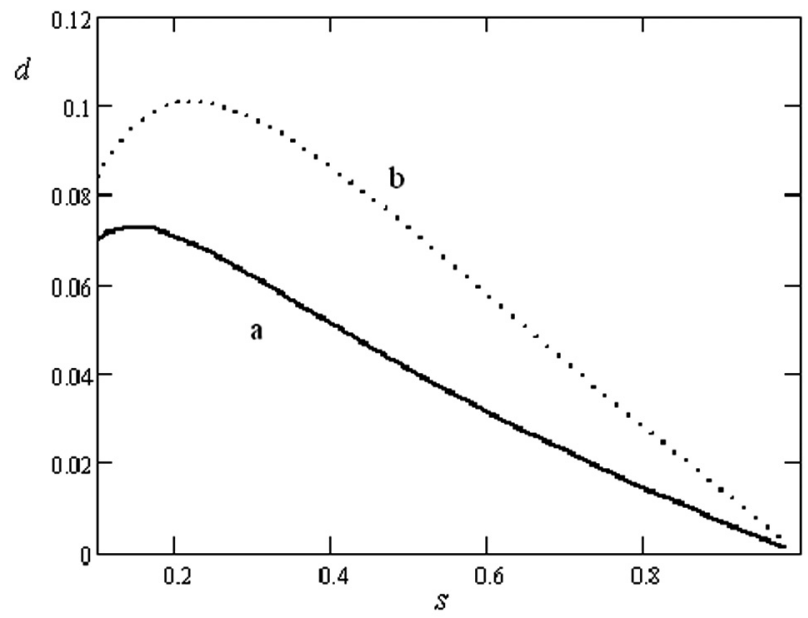

Fig. (8). Oscillation amplitude $d$ of relative supersaturation vs relative feeder width $s=l / L$ for feeding half-period $T$ equal to $1 \mathrm{~s}$ (a) and $2 \mathrm{~s} \mathrm{(b);} m=3, p=0.5$. 


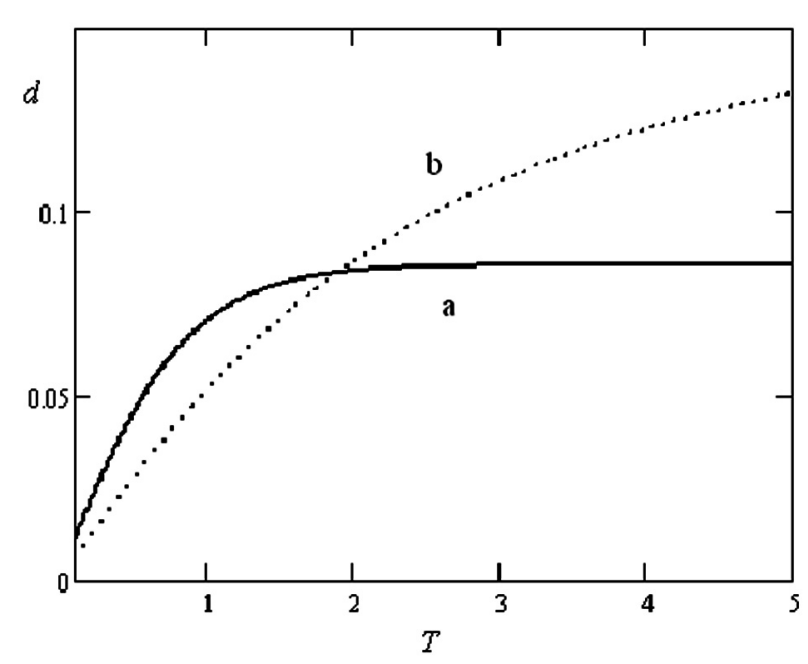

Fig. (9). Oscillation amplitude $d$ of relative supersaturation vs feeding half-period $T$ for relative feeder width $s=l / L=0.1$ (a) and $l / L=0.4(\mathrm{~b}) ; m=3, p=0.5$.

parameters, even in the simplest model of linear kinetics. This dependence is multiparametric. In the model under study it depends on four parameters: solution flow (pump capacity) $p l$, feeder swing period $T$, relative feeder width $s$ and flow velocity modulation depth $m$. The latter is unknown, as a rule, and depends on specific parameters of the growth device. The experimental situation is even more complicated. The solution flow geometry in the growth chamber and temporal characteristics of the flow depend also on the feeder height above the crystal, growth chamber height, and even on growth chamber material. Therefore, comparison of theoretical and experimental results may be only qualitative. This comparison will be based on the idea put forward in the introduction, according to which one of the most dangerous factors in terms of defect formation is periodic modulation of solution supersaturation on the growing crystal surface that leads [7] to development of the instability of the train of elementary steps, growing surface roughness and, as a consequence, to trapping of solution. Inclusions determine light scattering in a crystal corresponding to pronounced changes in the difference between the values of maximum and minimum supersaturations. Mean supersaturation defining mean growth rate (Fig. 6) changes insignificantly. The latter effect is insignificant in terms of defect formation in the growth region of interest to us.

One of the results worthy of special notice is the dependence of light scattering on solution flow velocity (Fig. 3). This behavior is in qualitative agreement with the theoretical result (Fig. 7) according to which the relative supersaturation amplitude $d$ decreases with increasing flow intensity $p$ in the experimental parameter region $(p \geq 0.5)$, which corresponds to an increased degree of growth regime kinetics. The data in Fig. (6) lead to a conclusion of practical importance, namely, for growing crystals of a satisfactory quality at the growth rate of about $0.5 \mathrm{~mm} / \mathrm{h}$, an average solution flow onto the crystal of $0.5 \div 1 \mathrm{1} / \mathrm{min} \cdot \mathrm{cm}^{2}$ should be provided.

It follows from the data in Figs. (6 and 8) that mean supersaturation slightly decreases with increasing relative feeding slit width $s$, whereas the relative oscillation amplitude $d$ varies strongly (when $\mathrm{s} \geq 0.1$ ) (Fig. 8). A similar situation occurs with variation of feeding period $T$. The relative supersaturation amplitude $d$ also reduces substantially with a decrease in the feeding period $T$ (Fig. 9). Therefore, to reduce the oscillation amplitude $d$ of relative supersaturation and hence the danger of rough step bunch formation, one should choose the minimum possible feeding period $T$ and the maximum relative feeder width $s$. Of course, the proposed model does not reflect complexity of real hydrodynamic and diffusion flows in the crystallizer; specific forms of the plots in Fig. (5) markedly depend on the geometry and size of a growth chamber.

Let us now pass over to comparison of the experimental (Fig. 4) and theoretical results. It should be noted that we could not change the period $T$ in the experimental data, which impedes the comparison. We can only consider the behavior of light scattering $I_{\text {scat }}$ as a function of feeder height $H$ above the growing surface and feeder width $l$. However, it should be taken into account that these effects are interrelated: as the feeder height $H$ increases, the jet "spreads" (Fig. 2). This case is analogous to the case of a wider feeder. Jet spread depends on its initial width, i.e., the feeder width $s$. For a narrow feeder, the jet spread within the same limits of height variation is stronger. According to the theoretical concepts, this effect results in improvement of feeding quality, i.e., in a smaller value of the oscillation amplitude of relative supersaturation on the growing surface (Fig. 8a). This leads to a decreasing dependence of light scattering on height $H$ for a narrower feeder $(2 \mathrm{~mm})$. On the other hand, if the height $H$ grows, the solution jet pressure close to the growing face declines, which is equivalent to loss of pump efficiency at the same feeder height. This effect leads to an increasing dependence of light scattering on height $H$ for a wide feeder $(16 \mathrm{~mm})$. For a feeder of average width $(8 \mathrm{~mm})$, these two effects are compensated; this results in a weak dependence of light scattering on height $H$ and is favorable for stability of the growth process. In the latter case (for the relative feeder width $s \approx 0.2$ ), we also observed smooth growth without visible interlayer transitions between crystal regions grown with changed growth parameters.

Thus, the results of experimental and theoretical studies show that light scattering in crystals (determined by growth "roughness") at periodic feeding depend on the period and duty cycle of feeding and on the feeder height above the crystal. These results are in qualitative agreement. To obtain a more precise and adequate description of the mentioned dependences, it is necessary to solve a more comprehensive problem, taking into account real geometry of a growth chamber.

We can draw the following practical conclusions from the results of the theoretical and experimental studies:

I. To grow crystals of a satisfactory quality at the growth rate of about $0.5 \mathrm{~mm} / \mathrm{h}$, an average solution flow onto the crystal of $0.5 \div 1 \mathrm{l} / \mathrm{min} \cdot \mathrm{cm}^{2}$ should be provided.

2. The flow depends on slit width and, evidently, can be minimized for the relative slit width $l / L \sim 0.2 \div 0.4$.

3. It is possible to choose a feeder with a slit width $(\sim 8 \mathrm{~mm}$ in the considered growth chamber configuration) such 
that the feeding mode will not depend on feeder height above the slit.

4. To conserve the crystal quality when passing over from growing small crystals to larger ones, the following similarity conditions: constant optimal mean flow per growing surface unit and relative slit width $s=l / L$ should be met.

Naturally, optimal feeding parameters depend on the growth chamber geometry and size as well. Therefore, the presented parameters obtained for crystal growth with a cross section of about $40 \times 40 \mathrm{~mm}^{2}$, give only rough estimates for larger growth devices. Further studies, both theoretical taking into consideration geometries of growth devices and experimental-technological ones are required.

\section{CONCLUSION}

The influence of periodicity of growing surface feeding in dynamic (high-rate) techniques of crystal growth from solutions on crystal quality has been considered. It is shown that periodic feeding leads to relaxation oscillations of diffusion layer thickness which, in turn, may distort morphology of the growing surface and give rise to defect formation in the crystal. This effect may be minimized by choosing optimal feeding parameters (frequency, duty cycle). The influence of hydrodynamic feeding conditions on the quality of a KDP crystal with the size of a growing (101) face of $40 \times 40 \mathrm{~mm}^{2}$ using high-rate profiled growth technique was investigated experimentally. Light scattering in a crystal is taken to be the parameter of quality. It is shown that light scattering in a growing crystal strongly depends on hydrodynamic conditions (solution flow, width and oscillation frequency of feeder, distance between the feeder and the growing face) and it is possible to choose parameters for which light scattering will be minimal. The results of the theoretical and experimental studies are in qualitative agreement and provide conditions for scaling growth devices.

\section{CONFLICT OF INTEREST}

Declared none.

\section{ACKNOWLEDGEMENTS}

Declared none.

\section{REFERENCES}

[1] Bespalov VI, Bredikhin VI, Ershov VP, Katsman VI, Lavrov LA. KDP and DKDP crystals for nonlinear optics grown at high rate. J Cryst Growth 1987; 82: 776-8.

[2] Bespalov VI, Bredikhin VI, Ershov VP, Zilberberg VV, Katsman VI. Gained experience in production of wide-aperture optical elements using KDP, DKDP crystal rapid growth technology. SPIE Proc 2000; 4424: 124-8.

[3] Zaitseva N, Carman L. Rapid growth of KDP-type crystals. Prog Cryst Growth Charact Mater 2001; (43): 1-118.

[4] Annenkov VI, Bespalov VI, Bredikhin VI, et al. Conversion of the 'Iskra-5' iodine laser to second-harmonic operating mode. Quantum Electron 2005; 35: 993-5.

[5] Andreev NF, Bespalov VI, Bredikhin VI, et al. A wide-aperture Pockels cell with three ring electrodes. Quantum Electron 2004; 34 : 381-4.

[6] Bredikhin VI, Ershov VP, Burenina VN, Mal'shakov AN, Potemkin AK. Possibility of increasing the optical breakdown threshold in KDP crystals. Quantum Electron 2007; 37: 489-94.

[7] Bredikhin VI, Galushkina GL, Kulagin AA, Kuznetsov SP, Malshakova OA. Competing growth centers and step bunching in KDP crystal growth from solutions. J Cryst Growth 2003; 259: 309-20.

[8] Bredikhin VI, Malshakova OA. Step bunching in crystal growth from solutions: model of nonstationary diffusion layer, numerical simulation. J Cryst Growth 2007; 303(1): 74-9.

[9] Bredikhin VI. Mass-transport and step bunching in crystal growth from solutions. Transp Theory Stat Phy 2008; 37: 504-19.

[10] Bredikhin VI, Galushkina GL, Kuznetsov SP. Schlieren technique to in situ monitor rapidly-growing KDP crystal surface. J Cryst Growth 2000; 219: 83-90.

[11] Bredikhin VI, Galushkina GL, Ershov VP, Rubakha VI, Shvetsova NR. Rapid growth of DKDP crystals from high-acidity solutions. J Cryst Growth 1999; 207: 122-6.

[12] Rashkovich LN. KDP-Family Single Crystals. Bristol: Adam Hilger 1991.

[13] Potapenko SYu. Two-dimensional mass transfer models for high-rate crystal growth from solution. J Cryst Growth 1993; 133: 132-40.

[14] Chernov AA. Modern Crystallography, v. III; Crystal Growth. Berlin: Springer 1984. 